\title{
Sal y salud, el punto de vista del consumidor Argentino obtenido por la técnica de grupos focales
}

\author{
Salt and health, Argentine consumers' views \\ via focus groups technique
}

\begin{abstract}
The consumption of high levels of salt $(\mathrm{NaCl})$ is associated with hypertension, and possible development of cardiovascular and cerebrovascular diseases. The aim of this study was to explore consumers' understanding of salt in their daily diet, their perceptions and knowledge about health implications and actions to reduce salt intake. Four focus groups were conducted (31 participants): one with university students, two with working adults and one older woman's group. Some participants were not aware of salt content of food whereas others demonstrated an awareness of salt in food, the role of salt as a preservative, affecting taste and health implications. Reducing consumption of salt tended to be a reactive event when personal/family health events took place rather than a proactive behaviour. Participants tended to consider their own consumption of salt as low/moderate but those who they lived with were perceived to have a high intake based only on table salt. The three adult focus groups expressed concerns about adding salt. Alternatives to salt such as seasonings/spices could be used as strategies to reduce its consumption. At the population level campaigns involving schools and mass media were suggested. Only university students raised the need to involve the food industry and food processors in reducing salt consumption. Keywords: sodium chloride, focus group, health, prevention, consumer.
\end{abstract}

\section{INTRODUCCIÓN}

La mayor parte de la sal (cloruro de sodio) que se ingiere ya se encuentra en los alimentos procesados. El resto proviene de la sal agregada durante la cocción y en la mesa y del agua de la cañería. La sal es uno de los ingredientes más utilizados en los alimentos procesados para mejorar sabor o textura (1). El sodio es un nutriente esencial que ayuda a regular el equilibrio hídrico del cuerpo, la transmisión nerviosa y los impulsos musculares. El exceso de sodio se excreta, pero los niveles excesivos de sodio retienen agua en el organismo. La ingesta de altos niveles de sal se asocia a hipertensión arterial (HTA), siendo éste el factor de riesgo más importante en el desarrollo de enfermedades cardiovasculares y accidentes cerebrovasculares (2). Existe una asociación significativa entre la ingesta de sal, la HTA y sus riesgos y el desarrollo de enfermedades vasculares (3-6).

El Reino Unido está tratando de reducir el consumo de sal de la población a través de políticas públicas de salud, porque
Marisa Beatriz Vázquez S.(1)

Silvia N. Lema R. (1)

Adriana Contarini C. (2)

Charlotte Kenten C. (3)

(1) Carrera de Nutrición, Facultad de Medicina, Universidad de Buenos Aires, Buenos Aires, Argentina (2) Departamento de Evaluación Sensorial, ISETA, Argentina (3) Dept of Primary Care and Public Health Sciences, King's College London, Inglaterra

Dirigir la correspondencia a: Profesora

Marisa $B$. Vázquez $S$ Marcelo T de Alvear 2202 - 4to piso - C1122AAH
Buenos Aires Argentin

Fono: 01145083865 Fax: 01145083859 E-mail:mbvazquez@fmed.uba.a!

Este trabajo fue recibido el 2 de Mayo de 2012 y aceptado para ser publicado el 5 de Septiembre de 2012.

el consumo actual supera el límite diario recomendado para los adultos. La presencia de sal "oculta" en alimentos procesados o comidas elaboradas fuera del hogar hace difícil para los individuos poder controlar o reducir su ingesta de sal (7-9).

En marzo de 2006 la Food Standards Agency (FSA) publicó objetivos voluntarios de reducción de la sal para los fabricantes de alimentos y minoristas (reducir la cantidad de sal en 85 categorías de alimentos procesados para el año 2010) (10). La industria alimentaria del Reino Unido ha adoptado los objetivos de reducción de sal propuestos por la FSA. The Food and Drink Federation (FDF) sostiene que el cambio debe ser gradual para permitir a los consumidores la adaptación gustativa (11). Estas estrategias desarrolladas para reducir la ingesta de sal, ubican al Reino Unido en situación más avanzada que a la Argentina en la temática. En julio de 2008, un estudio encargado por la FSA analizó la ingesta de sodio a través de orina de 24 horas en una muestra representativa de adultos entre 19-64 años. De acuerdo a él, el 
promedio de ingesta de sal para los adultos ha disminuido de 9.5 g en 2000 a 8.6 g en $2008(7,12)$. La FSA consideró que esta reducción se debe a la reformulación de productos y a cambios en el comportamiento de los consumidores (lectura de las etiquetas y menos agregado de sal discrecional). Según He y MacGregor la reducción de la ingesta de sal a 6 gramos por día equivale a una reducción del $13 \%$ en los accidentes cerebrovasculares y del $10 \%$ en la enfermedad isquémica del corazón en adulto (4).

En la Argentina, la reducción de la ingesta de sodio (máximo de $6 \mathrm{~g}$ diarios) ya fue tenida en cuenta en las Guías Alimentarias para la Población Argentina (GAPA) (13) diseñadas de acuerdo con los lineamientos del Instituto de Nutrición de Centro América y Panamá (14-16). Entre los 10 mensajes que las constituyen, las GAPA incluyen uno específico tendiente a la disminución de la ingesta diaria de sodio y pautas prácticas para su realización. Las Guías Alimentarias para la Población Infantil (GAPI) (17) también hacen recomendaciones para la promoción temprana de hábitos alimentarios preventivos de HTA. La Encuesta Nacional de Nutrición y Salud (ENNyS) realizada por el Ministerio de Salud de Argentina, reporta que sólo el 54,5\% de las mujeres de 18 a 49 años evaluadas presentaron valores normales de tensión arterial; en tanto que el resto presentó tensión arterial por encima de lo normal, en grado variable, con un comportamiento similar entre las diferentes regiones. No se estudiaron hombres en estos rangos de edad (18). Por otra parte, el Ministerio de Salud de la Nación lanzó en agosto de 2010 la campaña Menos Sal más Vida para disminuir el contenido de sodio en la producción de pan y prevé que la disminución de 1 gramo de sal en la dieta diaria podría evitar unos 20.000 eventos cardiovasculares por año y al menos 2.000 muertes $(19,20)$.

El Foro de la Organización Mundial de la Salud sobre la Reducción del Consumo de Sal en la Población reconoce que hay suficientes investigaciones científicas que relacionan la ingesta excesiva de sodio con varias enfermedades crónicas y en que la reducción del consumo de sal en la población es una de las formas más sencillas y costo-efectivas de reducir la HTA y otras enfermedades cardiovasculares. Recomienda basarse en tres pilares fundamentales: la reformulación de productos, la educación del consumidor y el desarrollo de entornos donde la elección de alimentos más sanos se vea facilitada para el consumidor (21).

Hay pocas investigaciones que reflejen las prácticas de ingesta de sal por parte de los consumidores. Estos han demostrado un reconocimiento insuficiente de su ingesta de sal ya que a través de un cuestionario los consumidores han demostrado no ser plenamente concientes de los riesgos potenciales de la alta ingesta de sal y los riesgos para la salud $(22,23)$.

Las prácticas alimentarias son consecuencia de la constelación de múltiples dimensiones individuales, socioculturales y económicas y del aprendizaje acumulado por las diferentes generaciones (24).

El conocimiento sobre la percepción que las personas tienen acerca de su consumo cotidiano de sal puede ser útil para el desarrollo de intervenciones orientadas a reducir la ingesta de sodio.

El equipo investigador se ha planteado una serie de interrogantes sobre el punto de vista del consumidor en relación con la ingesta de sal y su impacto en la salud. La técnica de grupos focales elegida para este estudio forma parte de la metodología de investigación cualitativa y utiliza la interacción de un grupo para explorar conocimientos, opiniones, experiencias y sistemas de valores; y para entender por qué las personas piensan y actúan de la manera que lo hacen (25-29).

El objetivo del estudio fue explorar la comprensión de la ingesta de sal en la alimentación diaria, las percepciones y los conocimientos sobre las implicancias para la salud y la opinión sobre acciones para reducir la ingesta de sal.

\section{SUJETOS Y MÉTODOS}

Procedimiento y participantes

La metodología elegida fue la técnica de grupos focales o grupos de enfoque.

El protocolo de trabajo fue aprobado por el Comité de Bioética del Hospital de Clínicas "José de San Martín" de la Universidad de Buenos Aires.

La convocatoria se realizó enviando una carta de invitación y una hoja informativa a personas seleccionadas por método de conveniencia. Se utilizó la base de datos de la Carrera de Nutrición, Facultad de Medicina, UBA; algunos participantes fueron sugeridos por miembros del equipo investigador.

Se elaboró una guía orientadora del debate con 4 categorías de información a explorar: hábitos alimentarios, ingesta de sal (presente y en el tiempo), implicancias del consumo de sal en la salud, e intervenciones / acciones para la salud (micro y macrosociales) (tabla 1).

Se hizo una prueba piloto con el propósito de testear las preguntas orientadoras del debate y cuestiones relacionadas con la modalidad de convocatoria y la técnica de coordinación.

Participaron del estudio 31 consumidores (4 hombres y 27 mujeres), mayores de 18 años. En la tabla 2 se presentan las características generales.

Se realizaron cuatro grupos focales (GF): el GF1, constituido por jóvenes estudiantes universitarios (no vinculados a la Carrera de Nutrición) residentes en la Ciudad Autónoma de Buenos Aires (CABA), los GF2 y GF4 estuvieron conformados por adultos en edad económicamente activa (el primero de CABA y el otro de la localidad de 9 de Julio, Provincia de Buenos Aires) y GF3, formado por mujeres de 52-73 años de 9 de Julio. Salvo en CABA -donde el nivel educativo fue casi exclusivamente terciario o universitario- en los dos grupos de 9 de Julio hubo proporciones similares de participantes con nivel educativo bajo (hasta secundario incompleto) y alto (desde secundario completo hasta terciario completo o universitario). Todos los participantes convivían en familia salvo 1 del GF3 que vivía solo.

Las reuniones grupales fueron coordinadas por un equipo constituido por dos investigadoras en el rol de monitor y observador. Al comienzo de cada reunión grupal se informaron los objetivos del estudio, los procedimientos para garantizar el anonimato y la dinámica del grupo de enfoque. Luego los participantes firmaron el formulario de consentimiento que incluyó autorización para grabar la sesión. Al término de cada sesión, los participantes completaron un cuestionario demográfico. Además, recibieron material educativo de las Guías Alimentarias para la Población Argentina y un viático por el tiempo invertido y el traslado. Para corroborar la validez, se realizó una síntesis oral del debate del grupo solicitando su acuerdo o aclaraciones y comentarios.

\section{Análisis de los datos}

Las grabaciones obtenidas en cada grupo de enfoque fueron transcriptas en su totalidad. Las transcripciones fueron leídas por el grupo de investigadores y codificadas por categorías de información (30). A los fines de preservar la confidencialidad de los datos, a los participantes involucra- 
dos se les asignó un número de código sólo conocido por las investigadoras. No se utilizó análisis estadístico por no ser aplicable a este tipo de estudio.

\section{RESULTADOS}

Del análisis de los resultados se desprende que los hábitos alimentarios de todos los grupos están dentro de las pautas culturales de la población urbana argentina.

\section{Categoría hábitos alimentarios}

Al menos la mitad de los participantes dijo consumir comidas rápidas como pizza o empanadas en los fines de semana, salvo el GF3 donde sólo un tercio de ellos lo manifestó. En $C A B A$, este consumo fue más frecuente y aparecieron también comidas pedidas a domicilio como sushi o comida mejicana. En 9 de julio las preparaciones caseras (incluso pastas) fueron más comunes y la expresión "comida rápida" se asoció con preparaciones o alimentos envasados más que con el concepto de delivery (envío a domicilio). Algunos dijeron consumir alimentos de conveniencia como: sopas cremas, postres, ravioles, salsas, puré de papas. En CABA manifestaron comprar alimentos de conveniencia como: milanesas de carne o pollo crudas listas para cocinar o tener en el freezer hamburguesas y trocitos de pollo.

Al indagar los motivos que predisponen al consumo de este tipo de comidas, aparecieron pocas respuestas, entre ellas el factor "tiempo". En tanto que lo que inclinó hacia lo elaborado en el hogar fue la idea de "más sano" o más confiable y la presencia de alguna patología:

...Yo, más que nada por el tiempo, la necesidad... (Mujer, 5057, ama de casa)

...Yo le digo: vos pensá que es más sano lo casero... (Mujer, +58 , comerciante)

...Sé lo que hago, sé lo que le pongo... (Mujer, 50-57, docente)
...En mi casa se come sin sal porque mi marido es hipertenso y mi mamá también... (Mujer, +58, ama de casa)

Algunos desconocían el contenido de sal de los alimentos o preparaciones. Otros la relacionaron espontáneamente con categorías diversas: contenida en alimentos, agregada durante la cocción o en el plato, como conservante, relacionada al gusto y asociada a HTA.

Identificaron como alimentos con sal a: productos de panificación, agua, agua mineral, comidas congeladas, gaseosas, embutidos, conservas, salsas preparadas, caldos comerciales, fiambres, queso, sándwich, conservas elaboradas a base de carne vacuna, aceitunas, chorizos, hamburguesas, salchichas, queso de rallar. Como alimentos elaborados sin sal mencionaron: tostadas, pan, caldos comerciales de verdura. Mencionaron a los envasados como alimentos que contienen sal. Manifestaron que la sal está presente en cantidad abundante en las preparaciones de delivery y en los lugares de comidas rápidas. Algunos integrantes identificaron a la sal como conservante.

Surgió espontáneamente la asociación entre el gusto y la presencia o ausencia de sal en los alimentos o preparaciones. Cuando los participantes manifestaron cocinar sin sal o con menos sal agregada siempre estuvo relacionado con la HTA de algún miembro del núcleo familiar. Además hubo una asociación general e inmediata (excepto grupo focal de jóvenes) con la sal agregada en la mesa y el temor por este hábito en hijos y nietos.

El GF1 en general dijo desconocer el contenido de sal de los alimentos o preparaciones. Sólo un participante del estudio refirió conocer el contenido de sal de los alimentos y preparaciones consumidas por leer la etiqueta de los paquetes y una integrante del GF3 dijo desconfiar de la veracidad de los rotulados:

...el tema de lo que se compra en paquete, uno mira la cantidad

TABLA 1

Guía orientadora del debate en grupos focales

Categorías

Hábitos alimentarios

Ingesta de sal (presente y en el tiempo)

Implicancias de consumo de sal en la salud

Intervenciones / acciones para la salud (micro y macrosociales)
Temas incluidos

Tipo de alimentos / estilos de comidas de consumo habitual Consumo de alimentos de conveniencia: frecuencia, oportunidad, motivos.

Conocimiento del contenido de sal en alimentos y preparaciones consumidos habitualmente. Fuentes de información.

Opinión sobre consumo habitual de sal.

Agregado o no de sal a preparaciones.

Percepción de la cantidad de sal consumida.

Evolución del consumo de sal a lo largo de la vida. Motivos.

Opinión sobre asociación entre alto consumo de sal y grupos poblacionales.

Conocimientos sobre repercusiones en la salud del consumo excesivo de sal.

Saberes acerca de la hipertensión arterial y otros efectos de la ingesta excesiva de sodio.

Conocimientos acerca de las recomendaciones de ingesta máxima de sal.

Opinión sobre acciones individuales posibles para la reducción de la ingesta de sal.

Conocimiento de campañas para la promoción de la salud basadas en la reducción de ingesta de sal.

Opinión sobre la campaña del Reino Unido para la reducción de contenido de sal en alimentos y preparaciones pre-elaborados.

Opinión y sugerencias para la implementación en nuestro país de campañas promotoras del cuidado de la salud basadas en la reducción de ingesta de sal. 
de sodio y a veces está especificado y no sabemos si es real o no... (Mujer, +58 , empleada)

Categoría ingesta de sal (presente y en el tiempo)

Con respecto a su consumo habitual de sal, en general fue percibido como moderado o bajo. La mayoría manifestó agregarla durante la cocción o en la mesa. Interrogados sobre cómo se daban cuenta de la cantidad de sal consumida sólo respondieron los integrantes del GF2, quienes la identificaron por la forma en que sus familiares usan el salero en la mesa.

En cuanto a la evolución del consumo, en general se manifestó una disminución a lo largo de la vida con excepción del GF4, en el cual más de la mitad de los integrantes refirió no haber tenido variaciones.

La mayoría de los participantes asociaron la disminución de su consumo de sal con la aparición de HTA propia y/o de algún familiar:

...creo que un poco el tema de los que dejan la sal es porque son hipertensos (...) porque si no la seguiría consumiendo... (Mujer, 34-41, ama de casa)

No hubo asociación de la ingesta excesiva con algún grupo poblacional en particular, con excepción de algunos participantes de 9 de Julio que la relacionaron con el género masculino.

Categoría implicancias del consumo de sal en la salud

$\mathrm{Al}$ indagar conocimientos sobre la relación consumo de sal / efectos sobre la salud, la mayoría de los participantes relacionó el consumo de cantidades importantes de sal con la HTA y en segundo lugar, con la retención de líquidos:

... yo sé que te hace mal, porque la abuela no podía comer mucha sal, pero muy bien no lo sé... (Mujer, 18-25, estudiante) ...yo sé que alguien con presión alta debe comer con menos sal... (Mujer, 18-25, estudiante)

... cuando se me hinchan las piernas, dejo la sal... (Mujer, +58, empleada)

Además, aunque en menor medida, apareció la necesidad de consumo de sal secundaria a baja presión arterial como conocimiento popular. En el GF2 nuevamente surgió la preocupación por el hábito observado en sus hijos de agregar sal en el plato. En el GF3 la asociación de menor ingesta de sal ante situaciones de HTA no apareció al indagar conocimientos sobre el efecto de la sal en la salud, sino cuando manifestaron haber variado el consumo de sal en el tiempo por HTA propia o de familiar conviviente.

\section{Categoría intervenciones / acciones para la salud (micro y macrosociales)}

Al preguntar sobre estrategias para reducir su ingesta de sal, predominó la propuesta de cocinar sin sal y apelar a alimentos condimentos y especias. Además, en el GF4 propusieron: que la reducción del consumo sea gradual; reducir el consumo de fiambres y consultar con un nutricionista para aprender cómo disminuir la ingesta. Sólo el GF2 mencionó la reducción de consumo de productos de panificación y su reemplazo por panificación sin sal. El GF1 propuso no acudir a locales de comidas rápidas; y tanto en éste como en el GF3 se propuso no consumir productos de copetín. Otras estrategias mencionadas fueron: uso de sales modificadas; alejar el salero del alcance; modificar la forma de cocción (vapor, cocinando las carnes sin trozar); elegir menos alimentos de conveniencia.

Sólo una persona mencionó como cantidad máxima diaria de sal recomendada 1 sobrecito de 2 gramos al día; los demás participantes se mantuvieron en silencio o manifestaron desconocer la recomendación.

La mayoría refirió no tener conocimiento de campañas para la promoción de la salud sobre la reducción de ingesta de sal. Sólo 2 participantes (GF2 y GF4) recordaron haber visto un logo relacionado con la salud cardiovascular y el contenido de sal/sodio de algún producto (agua envasada) y 4 participantes en los grupos de 9 de Julio hicieron referencia a un programa televisivo que mencionaba los riesgos del consumo excesivo.

Al poner en su conocimiento la iniciativa de la FSA del Reino Unido para la reducción de sal en productos alimenticios y solicitar sus opiniones, pocos participantes respondieron: la mayoría consideró de buena a muy buena la iniciativa, uno consideró que el gobierno no debería intervenir en decisiones individuales y otro opinó que el paladar latino prefiere comidas más sabrosas.

Sólo el GF2 respondió al interrogante sobre la factibilidad de una campaña similar en nuestro país: 1 persona opinó que es factible y que se podrían ofrecer condimentos sin agregado de sal, otra dijo que sería muy difícil y una tercera, que sería bueno dar esa opción.

Todos los grupos coincidieron en que es necesario generar conciencia sobre los riesgos del consumo excesivo de sal y propusieron que se hiciera mediante educación a padres y niños a través de la escuela y a través de campañas de difusión en medios de comunicación masiva.

En el GF1 (jóvenes universitarios) se propuso también la intervención con responsables de cadenas de comidas rápidas, industria alimentaria y elaboradores de preparaciones listas para consumir. Un participante sugirió cobrar la sal que se entrega en locales de consumo de alimentos.

Un participante del GF2 manifestó la necesidad de difundir la cantidad de sal/sodio contenido en los alimentos mediante carteles que grafiquen la proporción contenida ... tengo un esquema mental de cuántas calorías, cuánto puedo

TABLA 2

Características de los integrantes de los grupos focales

\begin{tabular}{|c|c|c|c|c|c|}
\hline \multicolumn{2}{|c|}{$\begin{array}{c}\text { Consumidores } \\
\text { discriminados por sexo }\end{array}$} & \multicolumn{2}{|c|}{ Trabaja } & \multicolumn{2}{|c|}{$\begin{array}{l}\text { Decisión sobre el } \\
\text { menú familiar }\end{array}$} \\
\hline Femenino & Masculino & SI & $\mathrm{NO}$ & $\mathrm{SI}$ & $\mathrm{NO}$ \\
\hline 6 & 1 & 0 & 7 & 0 & 7 \\
\hline 6 & 1 & 4 & 3 & 6 & 1 \\
\hline 9 & 0 & 4 & 5 & 9 & 0 \\
\hline 6 & 2 & 4 & 4 & 5 & 3 \\
\hline
\end{tabular}


sumar más o menos, del sodio también sería piola. Por ejemplo, un cartel que diga: con este paquete de galletitas te comiste toda la sal del día... (Mujer, 42-49, contadora)

\section{Categorías emergentes}

En el GF1 no aparecieron categorías adicionales a las previstas en el protocolo.

Los participantes de los otros grupos focales manifestaron de manera espontánea expresiones que no estaban contempladas en los temas o subtemas y que resultaron de interés para este estudio.

Algunos integrantes opinaron que actualmente hay más acceso a información relacionada con el cuidado de la salud en general y la conveniencia de restringir el consumo de sal en particular. Al mismo tiempo mencionaron como elementos asociados a la hipertensión, el estrés de la vida cotidiana y la falta de conciencia sobre los beneficios de prevenir enfermedades de manifestación no inmediata.

En GF2 y GF4 apareció la preocupación por la dificultad para tomar conciencia de problemas de salud cuyos síntomas y efectos no se perciben inmediatamente.

...mientras no nos cause ningún trastorno o un llamado de atención es como que no le damos bolilla... (Mujer, 50-57, ama de casa)

...no tomo conciencia que me hace mal. Es terrible, pero no me llega lo que dicen las chicas, no me llegan las palabras, no me Ilegan... (Mujer, 34-41, ama de casa)

Con excepción del GF1, en los demás grupos apareció la preocupación por el uso indiscriminado del salero en los niños, adolescentes y adultos jóvenes, con especial énfasis en aquellos integrantes decisores del menú familiar. Y se reiteró la falta de conciencia sobre los efectos a largo plazo.

...porque no hay, me parece, en los chicos (conciencia) que la sal es mala, yo nunca les dije. Les digo: "deja de ponerle tanta sal" porque es un desparramo, pero no les profundizo de por qué no tienen que ponerle tanta sal... (Mujer, 42-49, contadora) ...y como, aparentemente, no tienen un problema; y le ponen y le ponen y yo estoy siempre nena pará, pará... (Mujer, +58 años, ama de casa)

En GF2 y GF4 aparecieron espontáneamente referencias a indicaciones por parte de pediatras o médicos sobre la reducción del consumo de sal tanto en forma preventiva como ante eventos de enfermedad.

La escuela y el hogar fueron señalados por algunos integrantes como los ámbitos propicios para la generación de saberes relacionados con el cuidado de la salud.

\section{DISCUSIÓN}

El presente estudio ha explorado saberes, opiniones y percepciones en 4 grupos de personas con diferentes características, enfocando temas diversos como sus hábitos de consumo de sal, las razones con las cuales los explican y saberes y opiniones relativos al efecto de la sal en el organismo y a acciones preventivas de ingesta excesiva.

Varios alimentos y preparaciones cuyo consumo las GAPA y $\operatorname{GAPI}(13,17)$ recomiendan disminuir fueron identificados como de alto contenido de sal por muchos de los participantes, pese a lo cual manifestaron consumirlos con frecuencia variable y tenerlos en cuenta a la hora de proponer acciones de reducción de ingesta de sal.

Llama la atención el hecho de que la reducción del consumo de sal y/o de alimentos o preparaciones que la contienen ocurrió predominantemente como parte del tratamiento de eventos personales o familiares de salud y no como conducta preventiva. En algunos casos se hizo referencia a la dificultad para adoptar ese hábito preventivo aún entre quienes tenían familiares con HTA.

El desconocimiento sobre la ingesta máxima diaria recomendada de sal atravesó a todos los grupos, así como la percepción de que falta conciencia de los efectos nocivos a largo plazo que tendría superar esa recomendación. Pese a que la ingesta de alimentos de alto contenido de sal (comidas "rápidas", alimentos de conveniencia, productos de panificación, sopas y caldos comerciales, comidas enviadas a domicilio) fue referida en todos los grupos para todos los miembros convivientes (aunque con frecuencia variable), se observó la tendencia a considerar el propio consumo de sal como bajo a moderado y a detectar una ingesta alta en otros miembros convivientes, basándose sólo en la sal agregada en la mesa. Estos dos últimos hallazgos dan sustento a la recomendación del Foro de la Organización Mundial de la Salud sobre la Reducción del Consumo de Sal en la Población (21) relacionada con la necesidad de ofrecer entornos facilitadores de elecciones alimentarias saludables y de intervenciones de educación del consumidor.

El agregado de sal en la mesa por parte de "los otros" se manifestó como preocupación en los 3 grupos constituidos por adultos mayores de 26 años, la mayoría de los cuales eran decisores del menú familiar. Estos resultados coinciden con los obtenidos por Marshall, Bower y Schroder y por Purdy, Armstrong y Mcllveen $(22,23)$ en relación a la escasa percepción que las personas tienen sobre su consumo de sal y son consistentes con la sugerencia de algunos participantes de este estudio de informar al consumidor qué proporción de la cantidad máxima recomendada es la que aportan ciertos alimentos más allá de la sal de mesa.

Con relación a las campañas para la reducción de la ingesta de sal, fue común a todos los grupos el desconocimiento de su existencia, la recomendación de su lanzamiento y la inclusión de medidas que involucren a la escuela y los medios de comunicación. Resulta llamativo que sólo el grupo de jóvenes haya planteado la necesidad de involucrar a la industria alimentaria y a los elaboradores de comidas rápidas, comidas enviadas a domicilio y alimentos de conveniencia. Algunos integrantes plantearon que la reducción del contenido de sal debe ser gradual para favorecer la adaptación de las personas, concepto coincidente con la recomendación de la FDF (11) y que es conveniente tener en cuenta a la hora de iniciar acciones.

El presente estudio cualitativo ha demostrado que algunos participantes son conscientes de los efectos del exceso de sal, pero que son menos conscientes de la cantidad de sal que están ingiriendo, de los efectos a largo plazo que esto puede tener sobre su salud y de la relevancia de adoptar conductas preventivas. A partir de estos resultados las investigadoras se proponen desarrollar un cuestionario a través del cual encuestar una muestra representativa para corroborar patrones de consumo y percepciones.

\section{RESUMEN}

La ingesta de altos niveles de sal $(\mathrm{NaCl})$ se asocia a hipertensión arterial, enfermedades cardiovasculares y cerebrovasculares. Objetivo: explorar la comprensión de la ingesta diaria de sal, percepciones y conocimientos sobre sus implicancias para la salud y la opinión sobre acciones para su reducción. Se realizaron cuatro grupos focales (31 participantes): uno de jóvenes estudiantes universitarios, dos de adultos en edad económicamente activa y otro de mujeres de 52-73 años. Algunos desconocían el contenido de sal de alimentos/prepa- 
raciones; otros la identificaban como: contenida en alimentos, agregada en cocción/plato, como conservante, relacionada al gusto y asociada a hipertensión arterial. La reducción del consumo de sal y/o alimentos/preparaciones que la contienen ocurrió predominantemente ante eventos de salud, no como conducta preventiva. Se observó tendencia a considerar el propio consumo como bajo/moderado y a detectar una ingesta alta en otros miembros convivientes, basándose sólo en la sal de mesa. Los 3 grupos de adultos manifestaron preocupación por esto último. Predominó la propuesta de cocinar sin sal y usar alimentos condimentos y especias como estrategias de reducción. Sugirieron campañas y medidas que involucren escuelas y medios de comunicación masivos. Los jóvenes propusieron involucrar a la industria alimentaria y elaboradores de comidas rápidas y alimentos de conveniencia.

Palabras clave: cloruro de sodio, grupos focales, salud, prevención, consumidor.

Agradecimientos: Al Doctor Gene Rowe del Institute of Food Research, Norwich, UK por sus aportes para la realización de este estudio.

\section{BIBLIOGRAFIA}

1. Desmond E. Reducing Salt: A challenge for the meat industry. Meat Sci 2006; 74: 188-96.

2. SACN (Scientific Advisory Committee on Nutrition) Salt and Health. 2003. The Stationery Office, Norwich, United Kingdom. Disponible en: http://www.sacn.gov.uk/pdfs/ sacn_salt_final.pdf

3. MacGregor GA. Salt: blood pressure, the kidney, and other harmful effects. Nephrol Dial Transplant 1998.;13: 2471-9.

4. He FJ; MacGregor GA. How Far Should Salt Intake be reduced. Hypertension: J Am Heart Assoc 2003; 42: 1093-9.

5. Gilbert PA; Heiser G. Salt and health: the CASH and BPA perspective. British Nutr Found Bull 2005; 30: 62-9.

6. Karppanen $\mathrm{H}$; Mervaala E. Sodium Intake and Hypertension. Prog Cardiovasc Dis 2006; 49: 59-75.

7. FSA (Food Standards Agency). UK salt intake levels heading in the right direction. 2008. Disponible en: http:/ /www. food.gov.uk/news/pressreleases/2008/jul/sodiumrep08

8. FSA (Food Standards Agency). Salt Myths. 2008. Disponible en: http:/Www.salt.gov.uk/salt_myths.htm

9. FSA (Food Standards Agency). Why 6g? A summary of the scientific evidence for the salt intake target. Medical Research Council Human Nutrition Research, Cambridge 2005. Disponible en: http://www.salt.gov.uk/why6g0408. pdf

10. IFST (Institute of Food Science and Technology). Information Sheet: Salt. 2007. Disponible en: http://www.ifst. org/science_technology_resources/for_food_professionals/ information_statementes/19515/Salt.

11. FDF (Food and Drink Federation) Salt: industry efforts to make a difference 2008. Disponible en: http:/www.fdf. prg.uk/resources/salt-thefactsfinal1_2.pdf

12. NCSR (National Centre for Social Research). An assessment of dietary sodium levels among adults (aged 19-64) in the UK general population in 2008, based on analysis of dietary sodium in 24 hour urine samples. Disponible en: http:/ www.food.gov.uk/multimedia/pdfs/08sodiumreport.pd)

13. Asociación Argentina de Dietistas y Nutricionistas. Guías Alimentarias para la Población Argentina. Lineamientos Metodológicos y Criterios Técnicos. 2da. Edición. República Argentina 2004.

14. AADYND. Guías Alimentarias para la Población Argentina. Lineamientos Metodológicos y Criterios Técnicos. República Argentina: Asociación Argentina de Dietistas y Nutricionistas Dietistas, 2000.

15. Asociación Argentina de Dietistas y Nutricionistas. Guías Alimentarias para la Población Argentina. Lineamientos Metodológicos y Criterios Técnicos. 2da. Edición. República Argentina 2004.

16. Molina V: Guías Alimentarias en América Latina. Informe de la consulta técnica regional de las Guías Alimentarias. An Venez Nutr v.21 n.1 Caracas junio 2008

17. Longo E. Guías Alimentarias para la Población Infantil. Consideraciones para los equipos de salud. Ministerio de Salud y Ambiente de la Nación. República Argentina 2006.

18. ENNyS. Encuesta Nacional de Nutrición y Salud, Programa Materno Infantil en Salud, Ministerio de Salud de la Nación, República Argentina. 2007. Disponible en: http:// www.msal.gov.ar/htm/site/ennys/pdf/documento_resultados_2007.pdf

19. Instituto Nacional de Tecnología Industrial (INTI): Campaña Menos Sal, Más Vida. Disponible en: http://WwW.inti.gov. ar/sabercomo/sc93/inti4.php

20. Legowski B, Legetic B. How three countries in the Americas are fortifying dietary salt reduction: $A$ north and south perspective. Health Policy 2011; 102: 26-33.

21. OMS. Organización Mundial de la Salud. Reducción del consumo de sal en la población. Informe de un Foro y una reunión técnica de la OMS. París, 2007.

22. Marshall S; Bower JA; Schroder MJA. Consumer understanding of UK salt intake advice. Br Food J 2007; 109: 233-45.

23. Purdy J; Armstrong G; Mcllveen H. The influence of socioeconomic status on salt consumption in Northern Ireland. International Journal of Consumer Studies. 2002; 26: 7180.

24. Lema, S. Educación alimentaria nutricional en la Argentina en crisis. DIAETA (Buenos Aires) 2002; 21 (101): 36-39.

25. Kitzinger J. The methodology of focus groups: the importance of interactions between research participants. Sociol Health IIIn 1994; 16: 103-21.

26. Kitzinger J. Qualitative Research: Introducing focus groups. Br Med J 1995; 311: 299-302 (Published 29 July 1995).

27. FAO. Guía Metodológica de Comunicación Social en $\mathrm{Nu}$ trición. En: http://www.fao.org.ar/docrep/003/x6957s/ х6957200.HTM. Visitada 26-08-2010.

28. Krueger RA; Casey MA. Focus Groups: A Practical Guide for Applied Research. Thousand Oaks, CA. Sage, 1994.

29. Powell, R.A. and Single, H.M. Focus Groups. Int J Qual Health Care 1996; 8:499-504.

30. Rabiee F. Focus-group interview and data analysis. Proc Nutr Soc 2004; 63:655-60. 